\title{
Fiber tracts of the dorsal language stream in the human brain
}

\author{
Kaan Yagmurlu, MD, ${ }^{1}$ Erik H. Middlebrooks, MD, ${ }^{2}$ Necmettin Tanriover, MD, ${ }^{3}$ and \\ Albert L. Rhoton Jr., MD'1 \\ Departments of ${ }^{1}$ Neurological Surgery and ${ }^{2}$ Radiology, University of Florida, Gainesville, Florida; and ${ }^{3}$ Department of \\ Neurosurgery, Cerrahpasa Medical Faculty, Istanbul University, Turkey
}

\begin{abstract}
OBJECTIVE The aim of this study was to examine the arcuate (AF) and superior longitudinal fasciculi (SLF), which together form the dorsal language stream, using fiber dissection and diffusion imaging techniques in the human brain.

METHODS Twenty-five formalin-fixed brains ( 50 hemispheres) and 3 adult cadaveric heads, prepared according to the Klingler method, were examined by the fiber dissection technique. The authors' findings were supported with MR tractography provided by the Human Connectome Project, WU-Minn Consortium. The frequencies of gyral distributions were calculated in segments of the AF and SLF in the cadaveric specimens.
\end{abstract}

RESULTS The AF has ventral and dorsal segments, and the SLF has 3 segments: SLF I (dorsal pathway), II (middle pathway), and III (ventral pathway). The AF ventral segment connects the middle (88\%; all percentages represent the area of the named structure that is connected to the tract) and posterior $(100 \%)$ parts of the superior temporal gyri and the middle part (92\%) of the middle temporal gyrus to the posterior part of the inferior frontal gyrus ( $96 \%$ in pars opercularis, $40 \%$ in pars triangularis) and the ventral premotor cortex (84\%) by passing deep to the lower part of the supramarginal gyrus (100\%). The AF dorsal segment connects the posterior part of the middle (100\%) and inferior temporal gyri $(76 \%)$ to the posterior part of the inferior frontal gyrus ( $96 \%$ in pars opercularis), ventral premotor cortex (72\%), and posterior part of the middle frontal gyrus (56\%) by passing deep to the lower part of the angular gyrus (100\%).

CONCLUSIONS This study depicts the distinct subdivision of the AF and SLF, based on cadaveric fiber dissection and diffusion imaging techniques, to clarify the complicated language processing pathways.

http://thejns.org/doi/abs/10.3171/2015.5.JNS15455

KEY WORDS arcuate fasciculus; brain; fiber dissection; fiber tracts; language processing; superior longitudinal fasciculus; anatomy

$\mathrm{T}$ HE contemporary dual stream language model is analogous to the visual system, which is composed of ventral "what" and dorsal "where" streams. ${ }^{11,31}$ The ventral language stream is involved in semantic (meaning of word) processing of speech, and the dorsal stream controls phonological (word production) processing of speech. ${ }^{11,59}$ The arcuate (AF) and superior longitudinal fasciculi (SLF) are involved in the dorsal language stream, while the middle longitudinal (MLF), inferior longitudinal (ILF), inferior frontooccipital (IFOF), and uncinate fasciculi (UF) are associated with the ventral language stream (see Fig. 3C). 15,18,31,59

This study examined the components of the dorsal language stream (SLF and AF) using postmortem fiber dissection and DTI (diffusion tensor imaging) techniques (Figs. 1-3). These anatomical findings were compared with data from clinical-radiological analysis and intraoperative stimulation of white matter (Table 1).

The frontoparietal network, called the SLF, was verified and delineated as the dorsal SLF I, the middle SLF II,

ABBREVIATIONS AF = arcuate fasciculus; $B A=$ Brodmann area; DTI = diffusion tensor imaging; fMRI = functional MRI; IFOF = inferior frontooccipital fasciculus; ILF = inferior longitudinal fasciculus; MLF = middle longitudinal fasciculus; ROI = regions of interest; SLF = superior longitudinal fasciculus; SMA = supplementary motor area; $\mathrm{UF}=$ uncinate fasciculus.

SUBMITTED February 26, 2015. ACCEPTED May 14, 2015.

INCLUDE WHEN CITING Published online November 20, 2015; DOI: 10.3171/2015.5.JNS15455. 

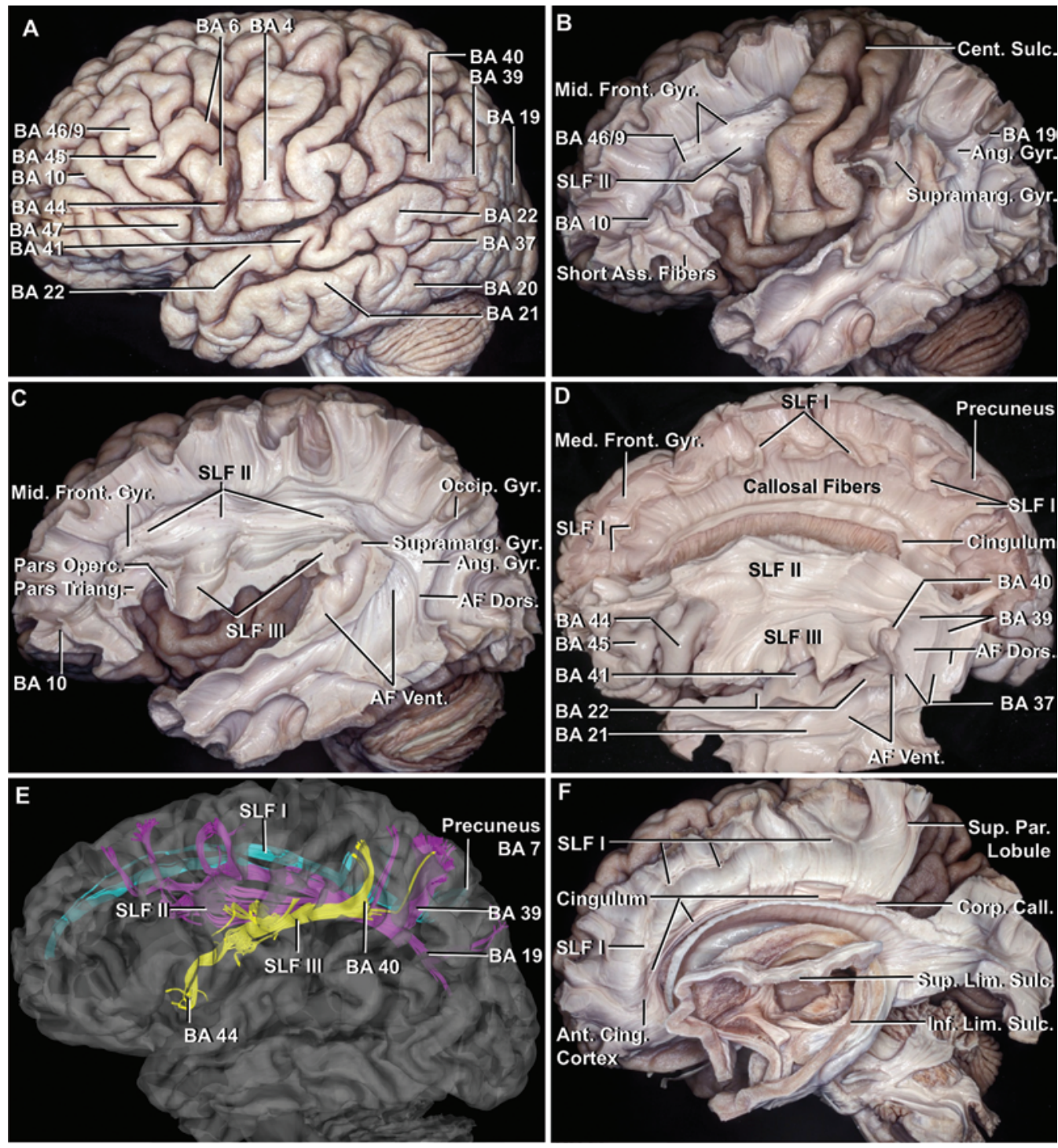

FIG. 1. A: Lateral view of the left hemisphere. The perisylvian cortex is composed of the ventral premotor cortex (BA 6), the inferior frontal gyrus (including the pars opercularis [BA 44], triangularis [BA 45], and orbitalis [BA 47]), the middle frontal gyrus (BA 6), the inferior parietal lobule (including the supramarginal [BA 40] and angular [BA 39] gyri), and the superior (BA 22, BA 41, and BA 42), middle (BA 21, BA 37), and inferior temporal (BA 20) gyri. B: The central lobe, formed by the pre- and postcentral gyri, was preserved. Removal of the cortical gray matter and adjacent short association fibers exposed the long association fibers. The cortical gray matter and the short and long association fibers are positioned in order, from lateral to medial. The SLF II extends from the angular gyrus (BA 39) or anterior part of the middle portion of the occipital lobe (BA 19) and connects to the middle frontal gyrus (BA 10 or BA 46/9). The SLF II is located deep to the anterior part of the occipital lobe, the inferior parietal lobe, the midlevel of the central lobule, and the middle frontal gyrus. C: The dorsal language stream (SLF and AF) is the most superficial long association fiber tract in the cerebrum. The SLF consists of frontoparietal connection fibers, and the AF consists of frontoparietotemporal connection fibers. The middle fiber pathway in the suprasylvian area is the SLF II, which connects the angular gyrus or middle portion of the occipital lobe to the middle frontal gyrus. The ventral fiber pathway in the suprasylvian area is the SLF III, which connects the supramarginal gyrus to the pars opercularis. D: The AF ventral and dorsal segments in the temporal lobe, and the SLF I, II, and III are exposed. The SLF I has been exposed on the contralateral side, because it is located in the medial part of the hemisphere. The SLF I courses within the upper bank of the cingulate sulcus above the cingulum and medial to the callosal fibers, and connects the precuneus to the medial frontal gyrus (medial side of the superior frontal gyrus) and anterior calcarine cortex. The SLF III, SLF II, and SLF I are positioned in that order from lateral to medial and ventral to dorsal. The AF ventral segment, extending from the middle (BA 22) and posterior parts (BA 22, BA 41, and BA 42) of the superior temporal gyrus and middle part of the middle temporal gyri (BA 21), passes through the lower part of the supramarginal gyrus (BA 40) and just medial to the SLF III in the frontoparietal operculum. The AF dorsal segment originates from the posterior part of the middle (BA 37) and inferior temporal gyri (BA 20), and passes through the lower part of the angular gyrus (BA 39) and just ventral to the SLF II. E: Segments of the SLF in DTI. The SLF II originates from the anterior part of the occipital lobe (BA 19) on this DTI. F: Lateral view. The SLF I running between the superior parietal lobule and anterior cingulate cortex travels medial to the callosal fibers above the cingulum. Ang. = angular; Ant. = anterior; Ass. = association; Call. = callosum; Cent. = central; Cing. = cingulate; Corp. = corpus; Dors. = dorsal; Front. = frontal; Gyr. = gyrus; Inf. = inferior; Lim. = limiting; Med. = medial; Mid. = middle; Occip. = occipital; Operc. = opercularis; Par. = parietal; Sulc. = sulcus; Sup. = superior; Supramarg. = supramarginal; Triang. = triangularis; Vent. = ventral. 
and the ventral SLF III pathways along the suprasylvian area. In addition, 2 segments of the AF were identified: the ventral and dorsal components.

\section{Methods}

Twenty-five formalin-fixed brains (50 hemispheres) and 3 adult cadaveric heads were examined. The arachnoid and surface vessels were removed from the brains. All specimens were frozen at $-16^{\circ} \mathrm{C}$ for 2 weeks..$^{40}$ Twenty-four hours after completion of the freezing process, the specimens were thawed with tap water, and the fiber tracts were dissected using microdissectors under $6 x$ to $40 x$ magnifications provided by a Zeiss Surgical Microscope (Carl Zeiss AG). Each stage of the dissections was recorded by 3D photography, as reported in a prior paper from this laboratory. ${ }^{63}$ We did not find a significant difference in the cortical connection areas of the SLF and AF between the right and left sides. Thus, we focused on the termination and originating areas on the cortex and the trajectory of each segment of the SLF and AF instead of on lateralization. The frequency distributions of the SLF and AF in the cortex were calculated based on postmortem cadaveric fiber dissection, independent of lateralization (right or left hemispheres).

Diffusion imaging from 2 healthy volunteers was analyzed. The scan data were provided, in part, by the Human Connectome Project, WU-Minn Consortium. MRI was acquired on a modified 3-T Siemens Skyra (Siemens AG). The diffusion sequence used a spin-echo echo-planar imaging (EPI) sequence with multiband EPI. The scan parameters were TR $5520 \mathrm{msec}$; TE $89.5 \mathrm{msec}$; flip angle $78^{\circ}$; refocusing flip angle $160^{\circ}$; FOV $210 \times 180$; matrix $168 \times 144$; slice thickness $1.25 \mathrm{~mm}$ (voxel size $1.25 \times 1.25$ $\times 1.25 \mathrm{~mm}$ ); multiband factor 3 ; and b-values 1000, 2000, and $3000 \mathrm{sec} / \mathrm{mm}^{2}$. Preprocessing consisted of B0 image intensity normalization, susceptibility distortion, eddy current, motion, and gradient-nonlinearity corrections, and registration with a T1-weighted 3D magnetizationprepared rapid acquisition gradient echo (MPRAGE) scan. All preprocessing used the freeware programs FreeSurfer (http://surfer.nmr.mgh.harvard.edu) and FSL (http://fsl. fmrib.ox.ac.uk). The preprocessed diffusion imaging data were imported into DSI Studio (http://dsi-studio.labsolver. org) for processing and tractography. The data were processed using generalized q-sampling imaging (GQI). Fiber tracking was then performed by seeding regions of interest (ROI) based on a priori knowledge from previous studies and fiber-tracking results from this study. ${ }^{23,41}$

\section{Results}

The SLF and AF are the most superficial long association fibers visible after removing the cortex (gray matter) and intergyral short $\mathrm{U}$ association fibers (Fig. 1B and C). Generally, the depth and length of the association fiber pathways are directly proportional; the longer association fibers that interconnect 2 distant areas are positioned at a deeper (more medial) level than shorter ones.

In the suprasylvian area, the main long association fiber pathway is the SLF, which has 3 parts: SLF I (dorsal), II (middle), and III (ventral). The SLF I (dorsal pathway) is positioned within the superior frontal gyrus, the SLF II (middle pathway) within the middle frontal gyrus, and the SLF III (ventral pathway) within the inferior frontal gyrus ${ }^{41}$ Starting at the lateral surface and moving medially, the components of the SLF are encountered in reverse order: SLF III, SLF II, and then SLF I (Fig. 1D). ${ }^{41}$

The SLF I, the dorsal fiber pathway, lies within the superior bank of the cingulate sulcus from the precuneus (medial surface of the parietal lobe) to the medial frontal gyrus (medial surface of the superior frontal gyrus), and travels just above the cingulum and medial to the callosal fibers. ${ }^{32,34,41,61,65}$ The SLF I connects the superior parietal lobule (Brodmann area [BA] 7) to the medial side of the superior frontal gyrus (BA 6, BA 8, BA 9) and anterior cingulate cortex (BA 32; Figs. 1D-F, 2A, and 2B). ${ }^{41,65}$

The SLF II, the middle fiber pathway, extends from the occipital lobe (BA 19 in 44\%; all percentages represent the area of the named structure that is connected to the tract) and/or the upper part of the angular gyrus (BA 39 in 56\%) to the middle part of the middle frontal gyrus (dorsolateral prefrontal cortex; BA 46/9 in 72\%) or the lateral frontopolar cortex (BA10 in 28\%). The SLF II passes deep to the cortex along the anterior part of the occipital lobe (BA 19), the inferior parietal lobe, the middle-third of the pre- and postcentral gyri, and the middle frontal gyrus (Fig. 1A-E). Fiber dissections corroborate DTI data, indicating that the SLF II is interrupted at the level of the precentral sulcus, where the fascicle is prominently grooved. This gutter along the SLF II most likely results from the presence of a vertical fiber pathway passing downward from the supplementary motor areas (SMAs) to the posterior part of the inferior frontal gyrus. In fact, Catani et al. ${ }^{8}$ have shown in multiple DTI studies that a short association tract, the fronto-aslant tract, runs between the premotor area, SMA, and pars opercularis.

The SLF III, the ventral fiber pathway, courses within the frontoparietal operculum and extends from the supramarginal gyrus (BA 40) to the pars opercularis (BA44). ${ }^{41,61,65}$

Another fiber pathway of the dorsal language stream is the AF, which connects Broca's area, located in the frontal lobe, and Wernicke's area, located in the temporal lobe. The AF is divided into ventral and dorsal segments according to its relationship with the supramarginal and angular parts of the inferior parietal lobe (Figs. 2 and 3).

The AF ventral segment extends from the middle (BA 22 in 88\%) and posterior (BA 22, BA 41, and BA 42 in $100 \%$ ) parts of the superior temporal gyrus and the middle part of the middle temporal gyrus (BA 21 in 92\%), always passes deep to the lower part of the supramarginal gyrus (BA 40 in 100\%) and medial to the SLF III (ventral pathway) in the frontoparietal operculum, and reaches the pars opercularis (BA 44 in 96\%), triangularis (BA 45 in 40\%), and ventral premotor cortex (BA 6 in 84\%).

The AF dorsal segment extends from the posterior part of the middle (BA 37 in 100\%) and inferior temporal gyri (BA 20 in 76\%), passes deep to the lower part of the angular gyrus (BA 39 in 100\%) in all hemispheres, and then travels ventral to the SLF II (middle pathway) to reach the pars opercularis (BA 44 in 96\%), ventral premotor cortex (BA 6 in 72\%), and posterior part of the middle frontal gyrus (BA 6 in 56\%). 

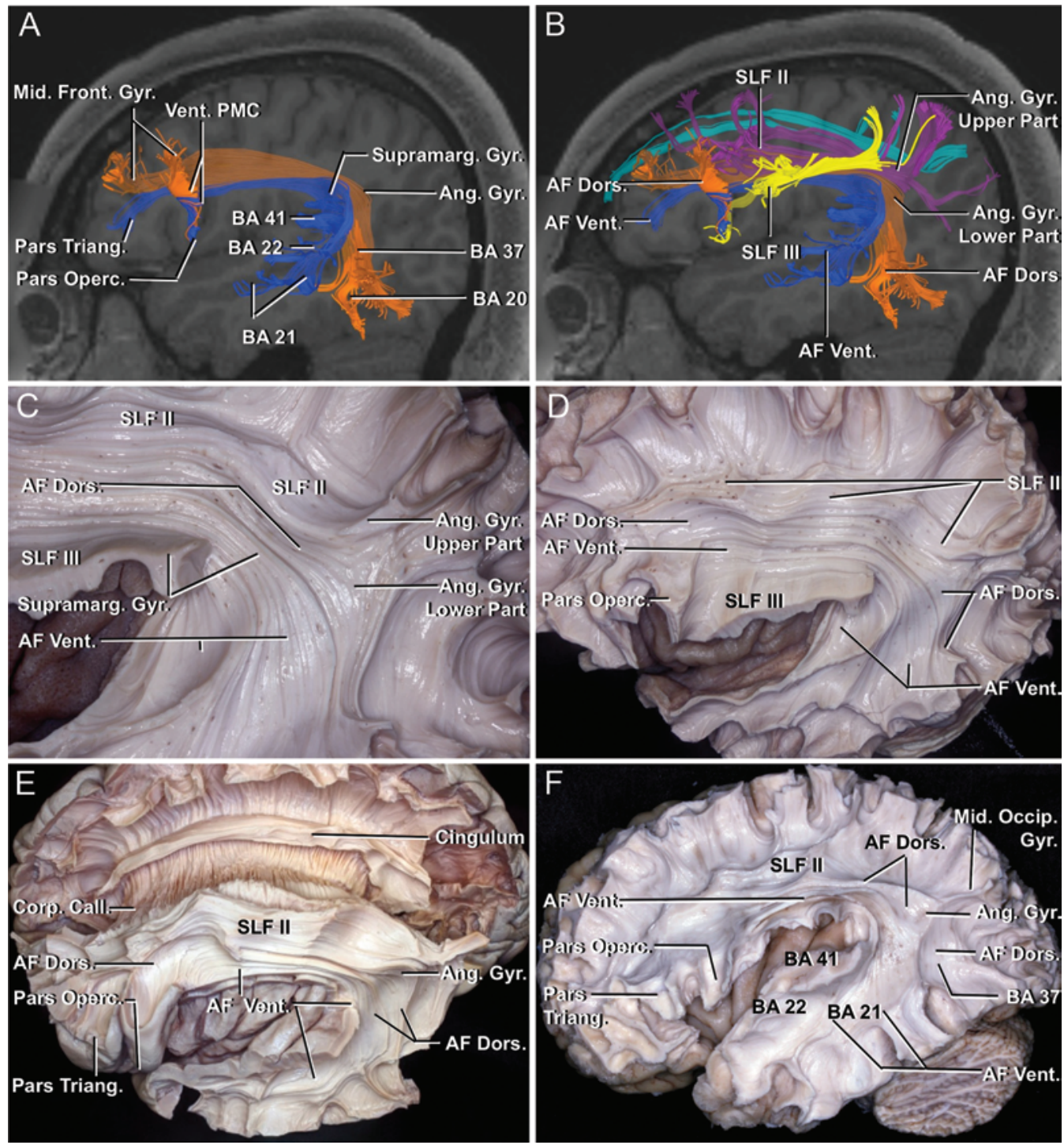

FIG. 2. A: Trajectory and termination of the AF segments on DTI. The ventral segment (blue) connects the middle (BA 22) and posterior parts of the superior temporal gyrus (BA 41) and the middle part of the middle (BA 21) temporal gyrus to the ventral premotor cortex and pars opercularis and triangularis by passing deep to the supramarginal gyrus. The dorsal segment (orange) connects the posterior part of the middle (BA 37) and inferior (BA 20) temporal gyri to the pars opercularis, ventral premotor cortex, and posterior part of the middle frontal gyrus by passing deep to the angular gyrus. B: Segments of the AF and SLF. After originating from the temporal lobe, the AF dorsal segment passes through the lower part of the angular gyrus and travels just ventral to the SLF II, which originates in the upper part of the angular gyrus or occipital lobe (BA 19). After arising in the temporal lobe, the AF ventral segment passes through the supramarginal gyrus and travels just medial to the SLF III in the frontoparietal operculum. C: Enlarged view of the inferior parietal lobule. The AF ventral segment passes through the lower part of the supramarginal gyrus and projects forward and medial to the SLF III in the frontoparietal operculum. The AF dorsal segment passes through the lower part of the angular gyrus, while the SLF II passes through the upper part of the angular gyrus. D: Relationship of the AF and SLF segments in the suprasylvian area. The AF ventral segment travels with the SLF III and medial to it, while the AF dorsal segment travels with the SLF II and ventral to it. E: The SLF III has been removed to expose the termination of the $\mathrm{AF}$ ventral and dorsal segments at the pars opercularis. F: The AF ventral and dorsal segments in another specimen. The SLF II also originates from the anterior part of the occipital lobe (BA 19) in this specimen. PMC = premotor cortex.

\section{Discussion}

\section{Fiber Tracts of the Dorsal Language Stream}

Until the latter part of the 20th century, the AF and SLF were considered to be part of the same fasciculus, connecting the posterior superior temporal gyrus (Wernicke's area) to the inferior frontal gyrus (Broca's area). ${ }^{61}$ In a cadaveric study, Türe et al. ${ }^{68}$ used SLF and AF as synonymous terms and noted that the SLF (AF) wraps in a $\mathrm{C}$ shape around the sylvian fissure and passes deep to the middle frontal gyrus, inferior parietal lobule, and middle temporal gyrus. More recently, studies in nonhuman primates have divided the SLF/AF into 4 separate components: SLF I, SLF II, SLF III, and AF. ${ }^{15,51,53,54}$ Makris et al. ${ }^{41}$ in a DTI-based study, showed the same 4 components in the human brain and suggested that the AF is a part of the SLF. Some cadaveric studies support the concept that the AF is a part of the SLF. ${ }^{22,44}$ In our study, we were able 

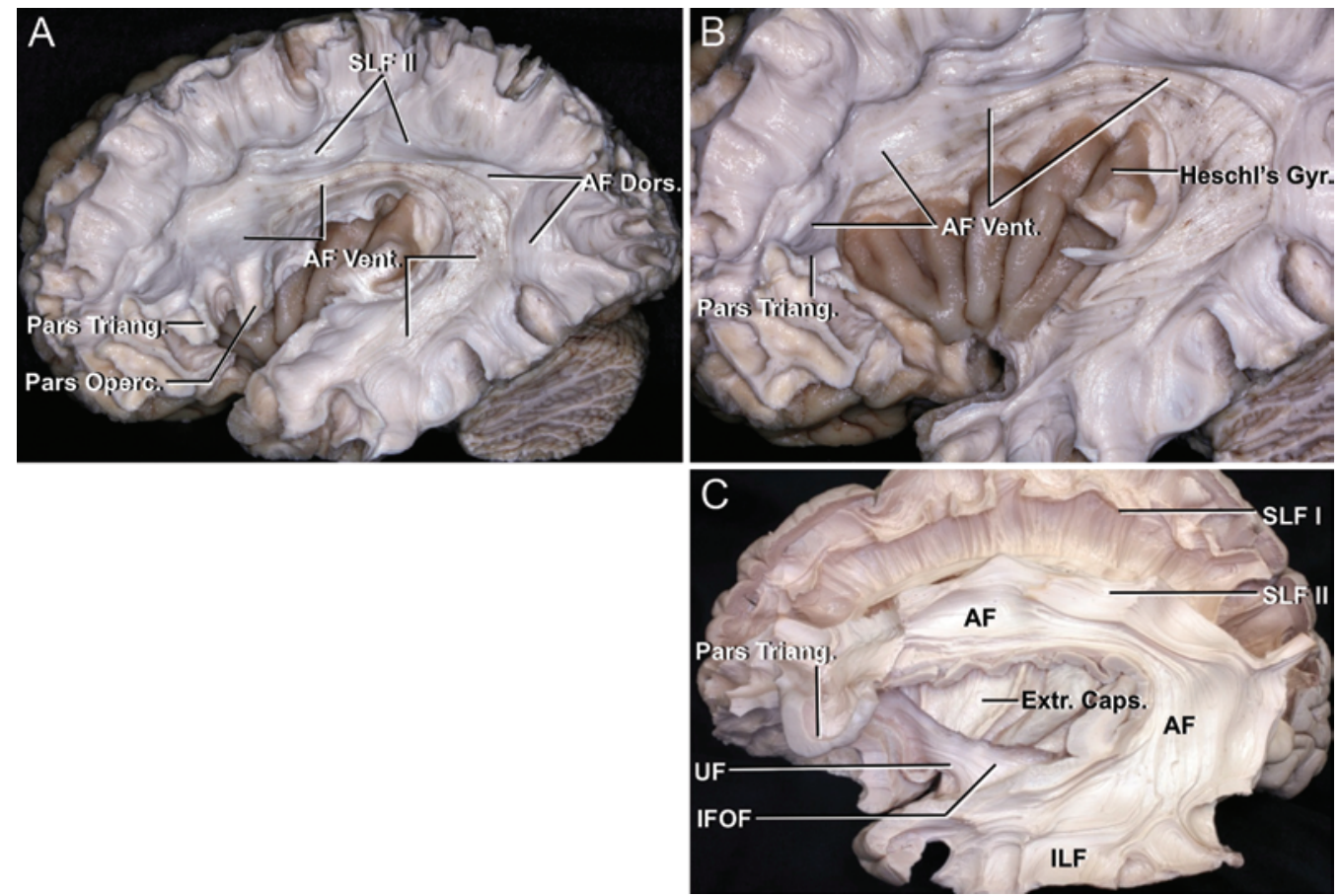

FIG. 3. A: The termination of the AF ventral segment at the pars triangularis and opercularis has been exposed. B: Further dissection. The posterior half of the pars triangularis has been removed to expose the termination of the AF ventral segment at the pars triangularis. C: Language processing ventral and dorsal streams. The dorsal stream consists of the AF and SLF, while the ventral stream consists of the IFOF, UF, ILF, MLF, and the extreme capsule. Extr. Caps. = extreme capsule.

to demonstrate the clear separation of 3 components of the SLF and 2 distinct parts of the AF through the fiber dissection technique and DTI.

\section{Segmentation of the Superior Longitudinal Fasciculus}

The SLF I connects the superior parietal lobe, important for recognizing limb and trunk location in body-centered coordinate space, with the premotor areas engaged in the higher aspects of motor behavior and with the SMA, which is important for intention and the initiation of motor activity. Therefore, the SLF I may be associated with higher-order control of body-centered action and the initiation of motor activity. ${ }^{41,53,61}$

The default mode network is the region of the brain that becomes activated when the brain is resting (but alert) and attention is focused on internal tasks such as memory retrieval and self-reflection. ${ }^{71}$ We suggest that the main subcortical connection of the default mode network, the long association bundle (SLF I) connected to the precuneus, may be to the anterior cingulate region. The precuneus plays a core role in self-consciousness with regard to the default mode network, which is activated during the "resting consciousness" state in which one does not engage intentionally in sensory or motor activity. In fact, lesions within the anterior cingulate cortex trigger an inability to detect errors, severe difficulty in resolving stimulus conflict, emotional instability, inattention, and even akinetic mutism, all of which may be completely or partly related to the default mode network..$^{10,71}$

As in our findings, the course of the SLF I has been previously defined by neuroimaging studies as running predominantly from the precuneus (medial BA 7) and less from the superior parietal lobule (BA 7) to the superior frontal gyrus (BA 8 and 9), SMA (BA 6), and anterior cingulate cortex by passing deep to the postcentral (BA 5) and precentral gyri (BA 4). ${ }^{41,53,61,65}$ In our study, during medial-to-lateral and lateral-to-medial fiber dissection, the SLF I was found to be the long association fiber tract located medial to the callosal fibers and above the cingulum in the superior bank of the cingulate sulcus.

The SLF I also has a close anatomical relationship with the cingulum in the medial side of the hemisphere. The cingulum fibers emanating from the medial cortex meet to form the cingulum above the corpus callosum within the cingulate gyrus. Therefore, cingulum fibers are oriented in anteroposterior, oblique, and superoinferior directions in the frontal lobe, and superoinferior and oblique directions in the parietal lobe. All cingulum fibers cross the cingulate sulcus to reach the cingulum within the cingulate gyrus. The SLF I, however, courses parallel to the cingulate sulcus, and it does not reach the cingulum.

However, 1 cadaveric study has claimed that the SLF I is not a frontoparietal long association fiber pathway, because the anterior portion of the SLF I is composed of short association fibers, while the posterior portion of the SLF I is composed of long association fibers. ${ }^{42}$ That study further noted that the precentral and central sulci run vertically across the cingulate sulcus to create the appearance of short association fibers in the anterior portion of the SLF I, while the long association fibers in the posterior portion of the SLF I belong to the SLF/AF. ${ }^{42}$ The long association fiber pathways located in the suprasylvian area have a wavy shape because of their intersection with perpendicular sulci on the convexity. Our finding, based 
TABLE 1. Fiber tract connections, functions, and associated disconnection syndromes

\begin{tabular}{|c|c|c|c|c|}
\hline \multirow{2}{*}{$\begin{array}{l}\text { Fiber } \\
\text { Tract }\end{array}$} & \multirow[b]{2}{*}{ Interconnected Regions } & \multicolumn{2}{|c|}{ Functional Role } & \multirow{2}{*}{$\begin{array}{l}\text { Disconnection } \\
\text { Syndromes }\end{array}$} \\
\hline & & Dominant Hemisphere & Nondominant Hemisphere & \\
\hline SLFI & Precuneus w/ ant cingulate cortex & $\begin{array}{l}\text { Regulation of higher aspect of } \\
\text { motor function; initiation of motor } \\
\text { activity;43,55,64 activation in resting } \\
\text { state; }{ }^{10,74} \text { integrate internally \& } \\
\text { externally driven information }{ }^{10,74}\end{array}$ & $\begin{array}{l}\text { Regulation of higher aspect of } \\
\text { motor function; initiation of motor } \\
\text { activity;43,55,64 activation in resting } \\
\text { state; }{ }^{10,74} \text { integrate internally \& } \\
\text { externally driven information } \\
10,74\end{array}$ & Unknown \\
\hline SLF III & $\begin{array}{l}\text { Supramarginal gyrus w/ pars } \\
\text { opercularis }\end{array}$ & $\begin{array}{l}\text { Articulatory aspects of lan- } \\
\text { guage }\end{array}$ & $\begin{array}{l}\text { Visuospatial attention, prosody, \& } \\
\text { music processing } 32,41,63,68\end{array}$ & $\begin{array}{l}\text { Articulatory disorders } \\
\text { (dysarthria/anar- } \\
\text { thria) }^{25,43,45,46,62}\end{array}$ \\
\hline AF ventral & $\begin{array}{l}\text { Mid \& pst part of the superior } \\
\text { temporal gyrus \& mid part of mid } \\
\text { temporal gyrus w/ ventral premo- } \\
\text { tor cortex, pars opercularis, \& } \\
\text { triangularis }\end{array}$ & $\begin{array}{l}\text { Phonological language process- } \\
\text { ing } 24,30 *\end{array}$ & $\begin{array}{l}\text { Phonological language process- } \\
\text { ing }\end{array}$ & $\begin{array}{l}\text { Phonological paraphasia } \\
\text { \& repetition disor- } \\
\text { der }{ }^{24,30}\end{array}$ \\
\hline AF dorsal & $\begin{array}{l}\text { Pst parts of the mid \& inferior } \\
\text { temporal gyri w/ ventral premotor } \\
\text { cortex, pars opercularis, \& pst } \\
\text { part of the mid frontal gyrus }\end{array}$ & $\begin{array}{l}\text { Lexical \& semantic language } \\
\text { processing }{ }^{24,30} \dagger\end{array}$ & $\begin{array}{l}\text { Prosodic activation of lan- } \\
\text { guage }{ }^{24,30} \mp\end{array}$ & $\begin{array}{l}\text { Transcortical motor } \\
\text { aphasia } 24,30\end{array}$ \\
\hline $\begin{array}{l}\text { Ant }=\text { anter } \\
* \text { Phonem } \\
\dagger \text { Lexical- }\end{array}$ & $\begin{array}{l}\text { id }=\text { middle; pst = posterior. } \\
\text { the smallest unit of sound in spoken I }\end{array}$ & age. & anings. & \\
\hline
\end{tabular}

on DTI and postmortem fiber dissection, showed that the short association fibers noted by Maldonado et al. ${ }^{42}$ in the anterior portion of SLF I are continuous with the posterior portion of the SLF I. As a result, we consider the SLF I to be a frontoparietal long association fiber pathway as has been stated in other studies in the literature..$^{32,34,41,61,65}$

The most prominent and easily perceived component of the SLF, the SLF II, is located in the middle part of the occipitoparietofrontal region. The SLF II shows right dominance in $65 \%$ of cerebra. ${ }^{41,65}$ Unlike previous reports, we also found a posterior extension of the SLF II to the occipital lobe (BA 19 in 44\%) and an anterior extension to the lateral frontopolar cortex (BA 10 in 28\%). The occipital extension of the SLF II supports the suggestion that an aberrant superior frontooccipital connection exists via the SLF II, although the concept of the superior frontooccipital fasciculus has already been ruled out through fiber dissection. ${ }^{69}$ Functionally, the SLF II can regulate the focusing of attention on different parts of space because of its course connecting the prefrontal cortex to the posterior parietal lobule. Likewise, damage to the SLF II is postulated to result in spatial working memory disorders such as neglect syndrome. ${ }^{65}$ Direct electrical inhibition of the SLF II during surgery causes a transient decrease in the appreciation of visual stimuli on the contralateral side. ${ }^{66}$

The SLF III courses between the supramarginal gyrus and the pars opercularis within the frontoparietal operculum. ${ }^{41}$ Functionally, the left SLF III is involved in phonological working memory, which is divided into a phonological store (in the left supramarginal gyrus) and an articulatory rehearsal module (in the left inferior frontal gyrus). Stimulation of the left SLF III produces articulatory disorders such as dysarthria or anarthria. ${ }^{24,43,44,59}$ The right SLF III, however, is involved in visuospatial attention, prosody, and music processing. ${ }^{30,39,60,65}$

\section{Segmentation of the AF}

In our study, the ventral AF originated in the middle and posterior parts of the superior temporal gyrus (BA 22, 41,42 ) and the middle part of the middle temporal gyrus (BA 21) and passed deep to the lower part of the supramarginal gyrus (BA 40). The dorsal AF originated in the posterior part of the middle and inferior temporal gyri (BA 37 and 20) and passed deep to the lower part of the angular gyrus (BA 39). After passing through the different parts of the inferior parietal lobe, the ventral AF continued ventrally with the SLF III, and the dorsal AF continued dorsally with the SLF II. Both segments terminated in the frontal lobe.

Some DTI and fiber-dissection studies have previously revealed a ventral and dorsal subdivision of the $A F^{23,28}$ Glasser and Rilling ${ }^{28}$ charted the superior and middle temporal gyrus pathways using DTI and functional neuroimaging. The superior temporal pathway, presumably the ventral AF in our study, connected the superior temporal gyrus (BA 22 and 42) to the inferior frontal gyrus (BA 44 and 6) and passed through the supramarginal gyrus. The middle temporal pathway, presumably the dorsal AF in our study, connected the middle temporal gyrus (BA 21 and 37) to the middle and inferior frontal gyri (BA 6, $9,45)$ and passed through the angular gyrus. In the study by Glasser and Rilling, the AF was found to be larger on 
the left side. A similar segmentation was suggested by Fernandez-Miranda et al., ${ }^{23}$ wherein the ventral or inner pathway connected the superior temporal (BA 22 and 42) and rostral middle temporal (BA 21) gyri to the pars opercularis (BA 44), and the dorsal or outer pathway connected the caudal middle temporal (BA 37) and inferior temporal (BA 20) gyri to the ventral premotor cortex (BA 6) and caudal middle frontal (BA 6 and 9) gyri. A comparison of our findings to those of Glasser and Fernandez-Miranda suggests that the classic AF model corresponds to the ventral AF in our study. ${ }^{23,28}$ Importantly, we focused in greater detail on the anatomofunctional relationship of the ventral and dorsal segments of the AF with each segment of the SLF.

Catani et al. ${ }^{9}$ also proposed a different classification for the AF based on a DTI study, separating the fascicle into superficial indirect and deep long segments. The indirect segment of the AF has been further divided into anterior (frontoparietal) and posterior (temporoparietal) parts. Some cadaveric studies have replicated this compartmentalization of the superficial fibers (indirect segment of Catani) into anterior and posterior parts. ${ }^{19,22,24,43,44}$, However, all studies have considered these fiber tracts to be part of the SLF rather than the AF. The AF ventral segment in our study corresponds to the classic AF.

In stimulation studies of the frontoparietal operculum, superficial (lateral) frontoparietal loop stimulation has resulted in dysarthria or anarthria, while deep stimulation has resulted in phonemic paraphasia (phonemic and repetition disorders). ${ }^{17,19,43,44}$ The superficial frontoparietal loop, which interconnects the supramarginal gyrus and pars opercularis, corresponds to the anterior part of the superficial segment of the AF as described by Catani et al., or more accurately, the lateral branch of the SLF/SLF III. ${ }^{9,41,44,61}$ In this vein, our findings also concur with previous studies that suggest the superficial (lateral) frontoparietal connection loop, or the anterior indirect segment of the AF as described by Catani, corresponds to the SLF III rather than the AF. $\cdot^{13,19,38}$

In our fiber dissections and DTI analysis, the SLF III and AF ventral segment were clearly separated along the frontoparietal operculum, with the SLF III located lateral to the AF ventral segment. Indeed, it has been shown that stimulation of the SLF III results in anarthria or dysarthria, while stimulation of the AF ventral segment (classic $\mathrm{AF}$ ) results in phonological (repetition) disorder. ${ }^{17,19,41,43,44}$

Makris et al. ${ }^{41}$ categorized the AF as part of the SLF system, arising in the caudal temporal region and traveling with the SLF II, which courses from the angular gyrus to the middle frontal gyrus in the suprasylvian area. What they referred to as the AF corresponds to the dorsal AF in our study, which travels just ventral to the SLF II.

\section{Connectivity and Functionality of the AF}

The traditional language model encompasses 3 cortical areas: Broca's, Geschwind's, and Wernicke's. Broca's area is located in the pars triangularis and opercularis in the left inferior frontal gyrus, Geschwind's territory is in the inferior parietal lobule, and Wernicke's area is in the posterior temporal area. ${ }^{15,52}$ "Extensive" Broca's and Wernicke's areas have been further suggested in DTI and functional studies. ${ }^{9}$ The extensive Broca's area includes the middle frontal and precentral gyri, in addition to the classic definition of the area. Studies of lesions, cortical stimulation, and neuroimaging have suggested separate language function subsystems in the inferior frontal cortex for phonological (basic sounds that make up words), syntactic (principles and processes by which sentences are constructed), and semantic (concepts and meanings of words) processing. The phonological region is located posteriorly in the inferior frontal gyrus at the border of the pars triangularis (BA 45) and pars opercularis (BA 44). ${ }^{5,12,21,33,37,56,64}$ The semantic region is located anterosuperiorly in the inferior frontal gyrus, pars orbitalis (BA 47) and triangularis (BA 45), and dorsolateral prefrontal cortex (middle part of the middle frontal gyrus) (BA 46/9).,18,25,35,46,47 Similarly, we found 4 frontal cortical areas included in the AF segments: the pars opercularis, pars triangularis, ventral premotor cortex, and the posterior part of the middle frontal gyrus. The ventral AF terminated in 3 areas: the pars opercularis (96\%), pars triangularis (40\%), and ventral premotor cortex (84\%). The dorsal AF terminated at the posterior part of the middle frontal gyrus (56\%), pars opercularis $(96 \%)$, and ventral premotor cortex (72\%). These findings support Catani's AF model, wherein the deep AF segment, which corresponds to the ventral segment of the AF, ends more anteriorly in the inferior frontal gyrus, and the AF superficial segment, which corresponds to the SLF III, ends more posteriorly (pars opercularis). ${ }^{9}$

The pars opercularis, and in particular the ventral premotor cortex, has been related to the occurrence of anarthria and/or dysarthria, and the pars triangularis, the frontal extension of the ventral AF (classic AF), has been implicated in the phonological processing of language.., 5 , 18,21,25,33,35,37,46,47,56,64 The ventral premotor cortex, which lacks plasticity in patients with low-grade gliomas, deserves special attention because both AF segments and the SLF III reach this area. ${ }^{72}$ Indeed, stimulation of the ventral premotor cortex, unlike any other opercular region, persistently results in anarthria because of its rich connections with the perisylvian subcortical fiber network.

The extended Wernicke's area includes the posterior part of the superior and middle temporal gyri (BA 22,37), the inferior parietal lobule (BA 39, 40), and the posterior temporal region. 1,9,20 The cortical termination areas we identified in the temporal lobe are the middle (BA 22) and posterior (BA 22, 41, 42) parts of the superior temporal gyrus, the middle and posterior parts of the middle temporal gyrus (BA 21 and 37), and the inferior temporal gyrus (BA 20). Functional studies suggest that the posterior part of the superior temporal gyrus (BA 22) is involved in phonological processing bilaterally, and the middle temporal gyrus (BA 21 and 37) is involved in lexical/semantic processing bilaterally, but prosody is exclusively processed

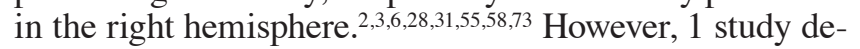
fines the extensive Wernicke's area as including the middle part of the superior temporal gyrus, which is involved in semantic language processing. ${ }^{14}$ Other DTI and functional MRI (fMRI) studies have bolstered claims that the middle temporal gyrus is a hub of ventral semantic fiber pathways and that it plays an important role in language comprehension. ${ }^{28,57,70}$ 
Geschwind's territory is composed of the supramarginal (BA 39) and angular (BA 40) gyri. Some recent direct cortical stimulation studies have supported the concept that the pars angularis is involved in lexical-semantic processing, while the supramarginal gyrus is more likely to be involved in phonetic working memory. ${ }^{28}$ Furthermore, others have suggested that the supramarginal gyrus is involved in articulation of speech and phonological processing, while the angular gyrus is involved in language perception, including syllable discrimination and identification. $17,36,44,61$

The ventral AF originates in the middle and posterior portions of the superior (BA 22, 41, 42) and middle (BA 21) temporal gyri (phonological and semantic processing areas), passes through the supramarginal gyrus (a phonological working memory area), and terminates in the ventral premotor cortex (BA 6) and pars opercularis (BA 44; another phonological processing area). The dorsal AF originates in the posterior part of the middle (BA 37) and inferior temporal gyri (BA 20; lexical-semantic processing areas), passes through the pars angularis (a semantic processing area), and terminates in the pars opercularis (BA 44) and ventral premotor cortex (BA 6; phonological areas) and posterior part of the middle frontal gyrus (BA 6 ; a semantic area).

Based on our dissections and published reports of neuroimaging, lesion-task-based, and direct electrical stimulation studies, the AF ventral segment is associated with phonological language processing, while the AF dorsal segment is associated with lexical and semantic language processing. ${ }^{23,28}$

As mentioned above, recent studies have also shown that stimulation of the deep part of the frontoparietal operculum (ventral AF) results in phonological paraphasia, while stimulation of the superficial part of the frontoparietal operculum (the location of the SLF III) results in dysarthria or anarthria. ${ }^{24,59}$ These findings also support our ventral and dorsal subdivision of the AF and the relationship between the AF ventral segment and SLF III in the frontoparietal operculum, based on their functional differences.

\section{Lateralization of the AF}

The structural correlates of language lateralization are not clear. Most evidence of language lateralization comes from studies of patients who have language deficits associated with brain lesions. In right-handed adults presenting with aphasia, the brain lesion is almost invariably located in the left hemisphere., ${ }^{7,26}$ Similar prevalence data have been found in patients undergoing brain surgery for epilepsy, with lateralization of language to the left hemisphere being observed in $90 \%$ of the right-handed patients. ${ }^{16,45,67}$ For instance, Geschwind found anatomical asymmetry in the size of the planum temporale, a region of the temporal lobe belonging to Wernicke's area, in $65 \%$ of the population. ${ }^{27}$ However, another study has recently found that left lateralization of the planum temporale does not correlate with language lateralization as assessed by the sodium amytal procedure. ${ }^{16}$ Several studies have reported a higher prevalence of leftward asymmetry of perisylvian white matter volumes, suggesting that language networks represent a more likely anatomical substrate for lateralization of language functions than cortical areas alone. ${ }^{16,25,29,48-50}$ Some neuroimaging studies that were based on the volume of fiber tracts and fMRI further noted the discrepancy that the ventral AF (superior temporal gyrus pathway of Glasser and Rilling or classic AF) was left lateralized, while the dorsal AF (middle temporal gyrus pathway of Glasser and Rilling) has no lateralization, meaning that the dorsal $\mathrm{AF}$ is the same on both sides ${ }^{23,28}$ However, most postmortem fiber dissection studies do not report a significant difference between the left and right $\mathrm{AF}^{44}$ In our study, we did not find a remarkable difference between the right and left sides in cortical connection areas of the SLF and AF. We focused on the connectivity and segmentation of the perisylvian network, which is composed of the SLF and AF, because of the unclear effect of cortical termination location and of AF/SLF volume on lateralization.

\section{Conclusions}

The dorsal language stream is formed by the AF and SLF. The AF has ventral and dorsal segments; the SLF has segments SLF I, II, and III or dorsal, middle, and ventral, respectively. ${ }^{23,28,41}$ Our study shows the relationship between these fiber tracts and depicts the distinct subdivision of the AF and SLF based on cadaveric fiber dissection and diffusion imaging studies. Although the SLF III (ventral pathway) and ventral AF are located in the frontoparietal operculum, they can be clearly separated based on fiber dissection and functional studies. The SLF II (middle pathway) and the dorsal AF can also be distinctly isolated in the suprasylvian area based on fiber dissection, neuroimaging, and functional studies. It is essential to better understand the complicated organization of language processing in the human brain.

\section{Acknowledgments}

We would like to thank the University of Florida Foundation for financial support. We would also like to thank Robin Barry for assistance in preparing the figures, Jessica Striley for editing and preparing the manuscript, and Marcelle Altshuler for searching the literature and doing preliminary editing.

\section{References}

1. Aboitiz F, García V R: The evolutionary origin of the language areas in the human brain. A neuroanatomical perspective. Brain Res Brain Res Rev 25:381-396, 1997

2. Binder JR, Frost JA, Hammeke TA, Bellgowan PS, Springer JA, Kaufman JN, et al: Human temporal lobe activation by speech and nonspeech sounds. Cereb Cortex 10:512-528, 2000

3. Binder JR, Frost JA, Hammeke TA, Cox RW, Rao SM, Prieto T: Human brain language areas identified by functional magnetic resonance imaging. J Neurosci 17:353-362, 1997

4. Bookheimer S: Functional MRI of language: new approaches to understanding the cortical organization of semantic processing. Annu Rev Neurosci 25:151-188, 2002

5. Caplan D, Alpert N, Waters G: Effects of syntactic structure and propositional number on patterns of regional cerebral blood flow. J Cogn Neurosci 10:541-552, 1998

6. Castillo EM, Simos PG, Davis RN, Breier J, Fitzgerald ME, Papanicolaou AC: Levels of word processing and incidental memory: dissociable mechanisms in the temporal lobe. Neuroreport 12:3561-3566, 2001 
7. Catani M, Allin MP, Husain M, Pugliese L, Mesulam MM, Murray RM, et al: Symmetries in human brain language pathways correlate with verbal recall. Proc Natl Acad Sci U S A 104:17163-17168, 2007

8. Catani M, Dell'acqua F, Vergani F, Malik F, Hodge H, Roy P, et al: Short frontal lobe connections of the human brain. Cortex 48:273-291, 2012

9. Catani M, Jones DK, ffytche DH: Perisylvian language networks of the human brain. Ann Neurol 57:8-16, 2005

10. Cavanna AE, Trimble MR: The precuneus: a review of its functional anatomy and behavioural correlates. Brain 129:564-583, 2006

11. Chang EF, Raygor KP, Berger MS: Contemporary model of language organization: an overview for neurosurgeons. J Neurosurg 122:250-261, 2015

12. Damasio H, Damasio A: Aphasia and the neural basis of language, in Mesulam M (ed): Principles of Behavioral and Cognitive Neurology. New York: Oxford University Press, 2000, pp 294-315

13. De Benedictis A, Duffau H, Paradiso B, Grandi E, Balbi S, Granieri E, et al: Anatomo-functional study of the temporoparieto-occipital region: dissection, tractographic and brain mapping evidence from a neurosurgical perspective. J Anat 225:132-151, 2014

14. DeWitt I, Rauschecker JP: Phoneme and word recognition in the auditory ventral stream. Proc Natl Acad Sci U S A 109:E505-E514, 2012

15. Dick AS, Tremblay P: Beyond the arcuate fasciculus: consensus and controversy in the connectional anatomy of language. Brain 135:3529-3550, 2012

16. Dorsaint-Pierre R, Penhune VB, Watkins KE, Neelin P, Lerch JP, Bouffard M, et al: Asymmetries of the planum temporale and Heschl's gyrus: relationship to language lateralization. Brain 129:1164-1176, 2006

17. Duffau H, Gatignol P, Denvil D, Lopes M, Capelle L: The articulatory loop: study of the subcortical connectivity by electrostimulation. Neuroreport 14:2005-2008, 2003

18. Duffau H, Gatignol P, Mandonnet E, Peruzzi P, TzourioMazoyer N, Capelle L: New insights into the anatomofunctional connectivity of the semantic system: a study using cortico-subcortical electrostimulations. Brain 128:797-810, 2005

19. Duffau H, Moritz-Gasser S, Mandonnet E: A re-examination of neural basis of language processing: proposal of a dynamic hodotopical model from data provided by brain stimulation mapping during picture naming. Brain Lang 131:1-10, 2014

20. Dronkers NF, Wilkins DP, Van Valin RD Jr, Redfern BB, Jaeger JJ: Lesion analysis of the brain areas involved in language comprehension. Cognition 92:145-177, 2004

21. Embick D, Marantz A, Miyashita Y, O’Neil W, Sakai KL: A syntactic specialization for Broca's area. Proc Natl Acad Sci U S A 97:6150-6154, 2000

22. Fernández-Miranda JC, Rhoton AL Jr, Alvarez-Linera J, Kakizawa Y, Choi C, de Oliveira EP: Three-dimensional microsurgical and tractographic anatomy of the white matter of the human brain. Neurosurgery 62 (6 Suppl 3):989-1028, 2008

23. Fernandez-Miranda JC, Wang Y, Pathak S, Stefaneau L, Verstynen T, Yeh FC: Asymmetry, connectivity, and segmentation of the arcuate fascicle in the human brain. Brain Struct Funct 220:1665-1680, 2015

24. Fridriksson J, Kjartansson O, Morgan PS, Hjaltason H, Magnusdottir S, Bonilha $\mathrm{L}$, et al: Impaired speech repetition and left parietal lobe damage. J Neurosci 30:11057-11061, 2010

25. Friederici AD, Meyer M, von Cramon DY: Auditory language comprehension: an event-related fMRI study on the processing of syntactic and lexical information. Brain Lang 74:289-300, 2000

26. Geschwind N, Galaburda AM: Cerebral lateralization. Bio- logical mechanisms, associations, and pathology: I. A hypothesis and a program for research. Arch Neurol 42:428 459,1985

27. Geschwind N, Levitsky W: Human brain: left-right asymmetries in temporal speech region. Science 161:186-187, 1968

28. Glasser MF, Rilling JK: DTI tractography of the human brain's language pathways. Cereb Cortex 18:2471-2482, 2008

29. Hagmann P, Cammoun L, Martuzzi R, Maeder P, Clarke S, Thiran JP, et al: Hand preference and sex shape the architecture of language networks. Hum Brain Mapp 27:828-835, 2006

30. Heilman KM, Watson RT: The disconnection apraxias. Cortex 44:975-982, 2008

31. Hickok G, Poeppel D: Dorsal and ventral streams: a framework for understanding aspects of the functional anatomy of language. Cognition 92:67-99, 2004

32. Jang SH, Hong JH: The anatomical characteristics of superior longitudinal fasciculus I in human brain: Diffusion tensor tractography study. Neurosci Lett 506:146-148, 2012

33. Just MA, Carpenter PA, Keller TA, Eddy WF, Thulborn KR: Brain activation modulated by sentence comprehension. Science 274:114-116, 1996

34. Kamali A, Flanders AE, Brody J, Hunter JV, Hasan KM: Tracing superior longitudinal fasciculus connectivity in the human brain using high resolution diffusion tensor tractography. Brain Struct Funct 219:269-281, 2014

35. Kaplan E, Naeser MA, Martin PI, Ho M, Wang Y, Baker E, et al: Horizontal portion of arcuate fasciculus fibers track to pars opercularis, not pars triangularis, in right and left hemispheres: a DTI study. Neuroimage 52:436-444, 2010

36. Kareken DA, Mosnik DM, Doty RL, Dzemidzic M, Hutchins GD: Functional anatomy of human odor sensation, discrimination, and identification in health and aging. Neuropsychology 17:482-495, 2003

37. Keller TA, Carpenter PA, Just MA: The neural bases of sentence comprehension: a fMRI examination of syntactic and lexical processing. Cereb Cortex 11:223-237, 2001

38. López-Barroso D, Catani M, Ripollés P, Dell'Acqua F, Rodríguez-Fornells A, de Diego-Balaguer R: Word learning is mediated by the left arcuate fasciculus. Proc Natl Acad Sci US A 110:13168-13173, 2013

39. Loui P, Alsop D, Schlaug G: Tone deafness: a new disconnection syndrome? J Neurosci 29:10215-10220, 2009

40. Ludwig E, Klingler J: Atlas Cerebri Humani. Boston: Little, Brown, 1956

41. Makris N, Kennedy DN, McInerney S, Sorensen AG, Wang $\mathrm{R}$, Caviness VS Jr, et al: Segmentation of subcomponents within the superior longitudinal fascicle in humans: a quantitative, in vivo, DT-MRI study. Cereb Cortex 15:854-869, 2005

42. Maldonado IL, Mandonnet E, Duffau H: Dorsal fronto-parietal connections of the human brain: a fiber dissection study of their composition and anatomical relationships. Anat Rec (Hoboken) 295:187-195, 2012

43. Mandonnet E, Duffau H: Understanding entangled cerebral networks: a prerequisite for restoring brain function with brain-computer interfaces. Front Syst Neurosci 8:82, 2014

44. Martino J, De Witt Hamer PC, Berger MS, Lawton MT, Arnold CM, de Lucas EM, et al: Analysis of the subcomponents and cortical terminations of the perisylvian superior longitudinal fasciculus: a fiber dissection and DTI tractography study. Brain Struct Funct 218:105-121, 2013

45. Mesulam MM (ed): Behavioral neuroanatomy: large-scale networks, associated cortex, frontal syndromes, the limbic system, and hemispheric specializations, in: Principles of Behavioral and Cognitive Neurology. New York: Oxford University Press, 2000, pp 1-120

46. Noesselt T, Shah NJ, Jäncke L: Top-down and bottom-up 
modulation of language related areas - an fMRI study. BMC Neurosci 4:13, 2003

47. Noppeney U, Price CJ: A PET study of stimulus- and taskinduced semantic processing. Neuroimage 15:927-935, 2002

48. Nucifora PG, Verma R, Lee SK, Melhem ER: Diffusiontensor MR imaging and tractography: exploring brain microstructure and connectivity. Radiology 245:367-384, 2007

49. Parker GJ, Luzzi S, Alexander DC, Wheeler-Kingshott CA, Ciccarelli O, Lambon Ralph MA: Lateralization of ventral and dorsal auditory-language pathways in the human brain. Neuroimage 24:656-666, 2005

50. Penhune VB, Zatorre RJ, MacDonald JD, Evans AC: Interhemispheric anatomical differences in human primary auditory cortex: probabilistic mapping and volume measurement from magnetic resonance scans. Cereb Cortex 6:661-672, 1996

51. Petrides M, Pandya DN: Association fiber pathways to the frontal cortex from the superior temporal region in the rhesus monkey. J Comp Neurol 273:52-66, 1988

52. Petrides M, Pandya DN: Distinct parietal and temporal pathways to the homologues of Broca's area in the monkey. PLoS Biol 7:e1000170, 2009

53. Petrides M, Pandya DN: Efferent association pathways originating in the caudal prefrontal cortex in the macaque monkey. J Comp Neurol 498:227-251, 2006

54. Petrides M, Pandya DN: Projections to the frontal cortex from the posterior parietal region in the rhesus monkey. $\mathbf{J}$ Comp Neurol 228:105-116, 1984

55. Poeppel D, Guillemin A, Thompson J, Fritz J, Bavelier D, Braun AR: Auditory lexical decision, categorical perception, and FM direction discrimination differentially engage left and right auditory cortex. Neuropsychologia 42:183-200, 2004

56. Poldrack RA, Wagner AD, Prull MW, Desmond JE, Glover GH, Gabrieli JD: Functional specialization for semantic and phonological processing in the left inferior prefrontal cortex. Neuroimage 10:15-35, 1999

57. Powell HW, Parker GJ, Alexander DC, Symms MR, Boulby PA, Wheeler-Kingshott CA, et al: Hemispheric asymmetries in language-related pathways: a combined functional MRI and tractography study. Neuroimage 32:388-399, 2006

58. Price CJ: The anatomy of language: contributions from functional neuroimaging. J Anat 197:335-359, 2000

59. Saur D, Kreher BW, Schnell S, Kümmerer D, Kellmeyer P, Vry MS, et al: Ventral and dorsal pathways for language. Proc Natl Acad Sci U S A 105:18035-18040, 2008

60. Schiff HB, Alexander MP, Naeser MA, Galaburda AM: Aphemia. Clinical-anatomic correlations. Arch Neurol 40:720-727, 1983

61. Schmahmann JD, Pandya DN: Fiber Pathways of the Brain. Oxford: Oxford University Press, 2006

62. Schmahmann JD, Pandya DN, Wang R, Dai G, D'Arceuil $\mathrm{HE}$, de Crespigny AJ, et al: Association fibre pathways of the brain: parallel observations from diffusion spectrum imaging and autoradiography. Brain 130:630-653, 2007

63. Shimizu S, Tanaka R, Rhoton AL Jr, Fukushima Y, Osawa S, Kawashima M, et al: Anatomic dissection and classic three- dimensional documentation: a unit of education for neurosurgical anatomy revisited. Neurosurgery 58:E1000, 2006

64. Stromswold K, Caplan D, Alpert N, Rauch S: Localization of syntactic comprehension by positron emission tomography. Brain Lang 52:452-473, 1996

65. Thiebaut de Schotten M, Dell'Acqua F, Forkel SJ, Simmons A, Vergani F, Murphy DG, et al: A lateralized brain network for visuospatial attention. Nat Neurosci 14:1245-1246, 2011

66. Thiebaut de Schotten M, Urbanski M, Duffau H, Volle E, Lévy R, Dubois B, et al: Direct evidence for a parietal-frontal pathway subserving spatial awareness in humans. Science 309:2226-2228, 2005

67. Toga AW, Thompson PM: Mapping brain asymmetry. Nat Rev Neurosci 4:37-48, 2003

68. Türe U, Yaşargil MG, Friedman AH, Al-Mefty O: Fiber dissection technique: lateral aspect of the brain. Neurosurgery 47:417-427, 2000

69. Türe U, Yaşargil MG, Pait TG: Is there a superior occipitofrontal fasciculus? A microsurgical anatomic study. Neurosurgery 40:1226-1232, 1997

70. Turken AU, Dronkers NF: The neural architecture of the language comprehension network: converging evidence from lesion and connectivity analyses. Front Syst Neurosci 5:1, 2011

71. Utevsky AV, Smith DV, Huettel SA: Precuneus is a functional core of the default-mode network. J Neurosci 34:932-940, 2014

72. van Geemen K, Herbet G, Moritz-Gasser S, Duffau H: Limited plastic potential of the left ventral premotor cortex in speech articulation: evidence from intraoperative awake mapping in glioma patients. Hum Brain Mapp 35:1587-1596, 2014

73. Vandenberghe R, Price C, Wise R, Josephs O, Frackowiak RS: Functional anatomy of a common semantic system for words and pictures. Nature 383:254-256, 1996

\section{Disclosures}

The authors report no conflict of interest concerning the materials or methods used in this paper or the findings specified in this paper.

\section{Author Contributions}

Conception and design: all authors. Acquisition of data: Rhoton, Yagmurlu, Middlebrooks. Analysis and interpretation of data: Rhoton, Yagmurlu, Middlebrooks. Drafting the article: Rhoton, Yagmurlu. Critically revising the article: all authors. Reviewed submitted version of manuscript: all authors. Approved the final version of the manuscript on behalf of all authors: Rhoton. Statistical analysis: Yagmurlu, Middlebrooks. Administrative/technical/ material support: Rhoton, Tanriover. Study supervision: Rhoton.

\section{Correspondence}

Albert L. Rhoton Jr., Department of Neurological Surgery, University of Florida, P.O. Box 100265, Gainesville, FL 32610. email: rhoton@neurosurgery.ufl.edu. 